\title{
Correction to: The Rapid Analysis of Antibiotics in Animal Meat and Egg Using a Novel SEP Method and UPLC-MS/MS
}

\author{
Jiang $\mathrm{He}^{1}$ - Lei Song ${ }^{2}$ - Guangyun Zhou ${ }^{2}$ - Lianwei Zhao ${ }^{2}$
}

Published online: 22 November 2017

๑) Springer-Verlag GmbH Germany, part of Springer Nature 2017

\section{Correction to: Chromatographia (2017) 80:1329-1342 https://doi.org/10.1007/s10337-017-3366-3}

In the original version of this article the affiliation 2 contained a mistake.

The corrected affiliation is given below.

Key Laboratory of Environmental Pollution Monitoring and Disease Control, Ministry of Education, School of Public Health, Guizhou Medical University, Guizhou, Guiyang 550025, China

Jiang $\mathrm{He}$

he6701324jiang@163.com

1 Center for Inspection and Testing for Quality and Safety of Guiyang Agricultural Products, Guiyang 550081, Guizhou, China

2 Key Laboratory of Environmental Pollution Monitoring and Disease Control, Ministry of Education, School of Public Health, Guizhou Medical University, Guiyang 550025, Guizhou, China 